\title{
SDN-based Joint Backhaul and Access Design for Efficient Network Layer Operations
}

\author{
Dongyao Wang, Efstathios Katranaras, Atta Quddus \\ Institute for Communication Systems \\ University of Surrey, Guildford, United Kingdom \\ \{w.dongyao, e.katranaras, a.quddus\}@surrey.ac.uk \\ Nikolaos Sapountzis \\ Mobile Communications Department \\ EURECOM, Biot, France \\ nikolaos.sapountzis@eurecom.fr \\ Luca Cominardi \\ IMDEA Networks Institute \& U. Carlos III of Madrid, \\ Madrid, Spain \\ luca.cominardi@imdea.org
}

\begin{abstract}
Small cell networks have been broadly regarded as an imperative evolution path for the next-generation cellular networks. Dense small cell deployments will be connected to the core network by heterogeneous backhaul technologies comprising fiber, microwave and high frequency wireless solutions, etc., which have their inherent limitations and impose big challenges on the operation of radio access network to meet the increasing rate demands in future networks. To address the above challenges, this paper presents an efficient design considered in the iJOIN (Interworking and JOINt Design of an Open Access and Backhaul Network Architecture for Small Cells based on Cloud Networks) project with the objective of jointly optimizing backhaul and radio access network operations through the adoption of SDN (Software Defined Networking). Furthermore, based on this framework, the implementation of several intelligent management functions, including mobility management, network-wide energy optimization and data center placement, is demonstrated.
\end{abstract}

Keywords-Small cell; backhaul; function split; software defined networking; energy efficiency; mobility

\section{INTRODUCTION}

Future mobile networks will have to provide an exceptionally greater traffic volume with diverse services such as machine-to-machine communication (low data rates) or 3D applications (high data rates). It is well-known that a very high system throughput of up to 500-1000 times today's throughput will be required by 2020 due to several drivers, e.g. the exponentially growing number of mobile internet users [1], more data-rich Internet content [2] and drastically increased mobile applications [3][4].

To cope with the exponentially growing traffic demand, small cells appear to be an option for mobile network evolution. As radio access points are placed closer to the user, the same spectrum could be reused to increase tremendously the overall capacity of the network. On the other hand, the technique of centralized processing could be applied to handle the increasing interference in very dense networks, to reduce energy-consumption, and to deploy and manage cellular networks in a cost-efficient way.

\author{
Fang-Chun Kuo, Peter Rost \\ NEC Europe Labs, \\ Heidelberg, Germany \\ \{fangchun.kuo, peter.rost $\} @$ neclab.eu \\ Carlos J. Bernardos \\ University Carlos III of Madrid, Madrid, Spain \\ cjbc@it.uc3m.es \\ Ignacio Berberana \\ Telefónica I+D S.A.U., Don Ramón de la Cruz \\ Madrid, Spain \\ ibfm@tid.es
}

The mobile network consists of the radio access network and the backhaul network which connects radio access and core network. So far, most radio access designs (including 3GPP (the 3rd Generation Partnership Project) architecture [6]) consider the backhaul network to be sufficiently dimensioned (over-provisioned). While this is already challenging in today's backhaul networks, the backhaul requirements will increase correspondingly as we move towards small cells and more centralized operation. In fact, in current mobile networks all user traffic is anchored to a central entity (the mobility anchor) which poses scalability issues and leads to sub-optimal paths between the users and their communication peers. This has triggered the study of how to provide mobility services in a more flat network architecture, enabling local access to the Internet, in order to reduce the amount of traffic traversing the core. Therefore, the limited backhaul resources must be considered when operating the radio access network and jointly managing access and backhaul network functionalities would be necessary.

In more recent publications [7][8][9], the iJOIN project proposed to support a flexible functional split by means of the RANaaS (RAN as a Service) infrastructure. RANaaS allows the execution of radio interface functionalities with different levels of centralization, and it provides the possibility to codesign and co-optimize the radio access and backhaul network. In this paper, we present the SDN-based framework considered in iJOIN. It centralizes the network control within a SDN controller which has the ability of fast response to dynamic network environments. Thus, it can realize an efficient interworking and joint optimization of radio access and backhaul network. Besides, practical implementations of three typical network operations based on this framework are analyzed and designed.

\section{SYSTEM ARCHITECTURE}

One of the key ideas of iJOIN is to centralize part of functionalities usually processed in an eNodeB (eNB) into the RANaaS platform. A RANaaS instance is composed of general purpose cloud-computing and storage infrastructure resources, similar to IaaS (Infrastructure as a Service) 
platform. Compared to the C-RAN (Centralized RAN) approach [5], the iJOIN project supports a flexible functional split, which allows for the execution of radio interface functionalities with different levels of centralization.

In RANaaS, the centralized radio access network (RAN) functionalities are implemented on a virtualized cloudinfrastructure. In addition, partial centralization is envisioned in order to cope with non-ideal backhaul characteristics, i.e. throughput and latency. Therefore, RAN centralization can be applied to heterogeneous backhaul networks.

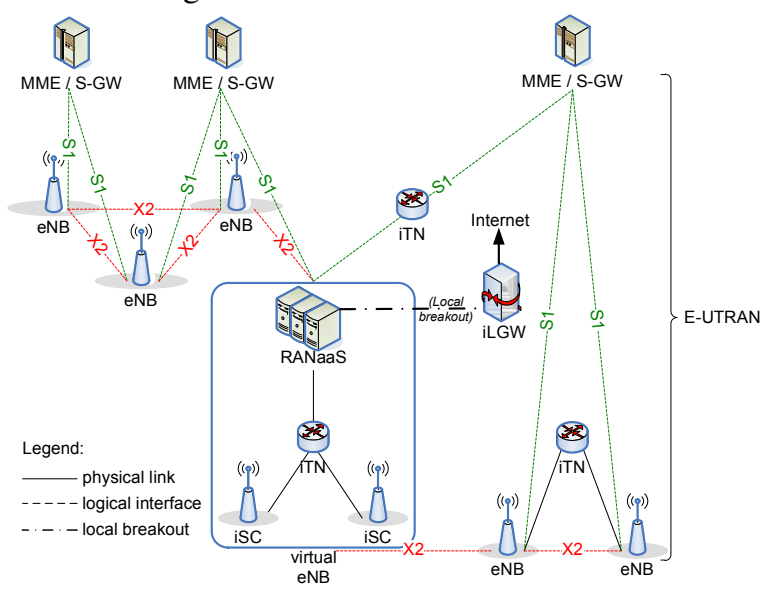

Fig. 1 iJOIN architecture

With the introduction of the RANaaS concept, the conventional 3GPP LTE/LTE-A architecture [6] can be extended as illustrated in Fig. 1. To support the functional split envisaged within the project, a new type of small cell is introduced: iJOIN Small Cell (iSC). These low power flexible radio access points implement all or part of the lower OSI (Open Systems Interconnection) layer(s) $(\mathrm{RF} / \mathrm{L} 1 / 2 / 3)$ that are traditionally executed at the base station. Furthermore, multihop links are possible from an iSC towards the RANaaS entity, the core network or another iSC via iJOIN Transport Node (iTN) which performs some kind of relay functionality on data plane. Additionally, for traffic selection and offloading needs, the iJOIN Local Gateway (iLGW) is introduced to behave as the local breakout point, and its functionality is close to the local gateway used in femtocells for LIPA (Local Internet Protocol Access) purposes.

The support of the flexible functional split provided by RANaaS has significant implications for the network layer:

- Different functional splits result in different requirements on the transport infrastructure that connects centralized and distributed functionalities. Thus, minimum required capacity and latency may significantly differ depending on the chosen functional split. This aspect can significantly influence the design and operation of the network.

- The implementation of the RANaaS concept allows for the support of virtual eNodeBs (veNB). In general, a veNB can be considered as the combination of one RANaaS entity and multiple iSCs connected to this instance. This architecture facilitates the implementation of complex cell cooperation techniques.

A co-design and interworking of radio access network and a heterogeneous backhaul network is expected to combat the challenges which are imposed by RANaaS. The following section presents the proposed framework which has been designed for supporting partly centralized RAN.

\section{SDN-BASED FRAMEWORK FOR JOINT OPERATION}

In the iJOIN project, a logically centralized approach is proposed based on SDN concept to orchestrate, coordinate and execute different network mechanisms. The technique of SDN as a new networking paradigm is currently attracting significant attention from both academia and industry. It allows for making currently rather static networks more flexible by tailoring and optimizing specifically them for different use cases, which means that a dynamic network environment could be created to adapt to the needs of the applications running on top of it [10].

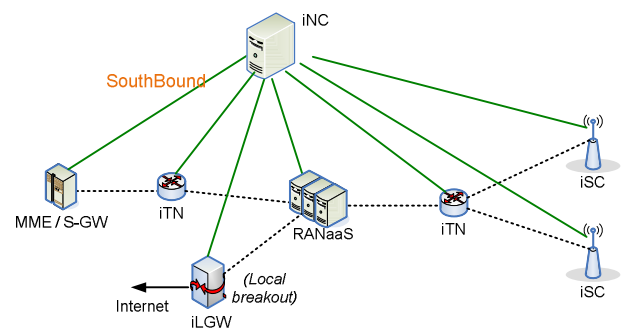

Fig. 2 SDN-based architecture and Network Controller

The basic framework designed for joint operation is shown in Fig. 2. In this architecture, a network entity called iJOIN Network Controller (iNC) is defined. The iNC controls both the radio access and the backhaul network through a SDN protocol. Thus, the iNC entity plays a key role in terms of configuring, monitoring and driving the operation of the rest of the RAN and backhaul network entities.

In compliance with the principle of the SDN concept, an iJOIN-extended OpenFlow (iOpenFlow) controller, located at the iNC, takes care of all the protocol interactions with the rest of the network entities. These entities only need to support the OpenFlow protocol using the so-called Southbound protocol interface. Moreover, inside the iNC exist multiple intelligent modules which support different management functions and are logically centralized. In the iJOIN project, several network layer operations are investigated, including the mobility support and its required signalling efforts, energy optimizations, admission and congestion control, load balancing and traffic routing. Fig. 3 illustrates the internal structure of the iNC with several such modules, designed to support network orchestration.

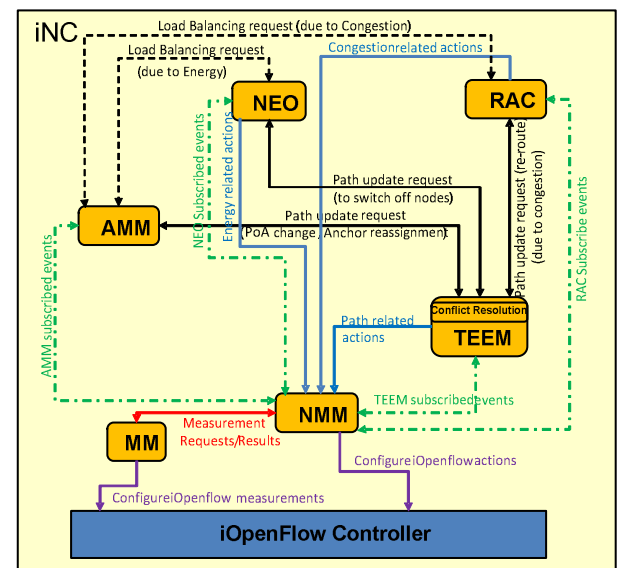

Fig. 3 Illustrated functional architecture

In the following, the individual modules are explained in further detail:

- Anchor and Mobility Management (AMM). This module implements the selection of the proper anchors 
on per-application and per-UE basis, and it ensures that sessions receive mobility support if needed. AMM is in charge not only of providing mobility support on an address (application) basis, but also to ensure that resources are optimally exploited, both in backhaul and radio access. This is achieved by selecting and using an anchor closer to the UE (User Equipment). This does not mean that for some flows legacy EPS (Evolved Packet System) Rel-10 mobility mechanisms (and anchors, i.e., the P/S-GW (Packet/Service GateWay)) are not used but that they are actually complemented by the iJOIN solutions.

- Network-wide Energy Optimizer (NEO). This module monitors the network status and load. It further runs intelligent algorithms to optimise the overall energy consumption while ensuring that the network wide performance is not compromised. NEO is in charge of taking network wide decisions about switching on/off physical nodes, as well as ensuring that UE traffic is still properly routed.

- Routing and Congestion (RAC). This module is in charge of avoiding network congestion in the radio access and backhaul network by properly configuring the network and requesting changes on the paths used by active data flows.

- Traffic Engineering Enforcement Module (TEEM). This is a key module that hosts all the intelligence required to compute the best path within the backhaul to support the different traffic and network-wide requirements. It further provides the necessary conflict resolution functions, e.g., to ensure that a request from the NEO module does not introduce congestion or contradicts a previous request from the RAC module.

- Measurement Module (MM). This module is in charge of configuring the iOpenFlow controller to perform the required measurements. These measurements might be dynamically requested by other modules. Envisioned metrics comprise locally experienced congestion, available neighbours, available data rates, and number of connected UEs. MM will support several reporting modes, e.g., asynchronous, periodic or event based.

- Network Model Module (NMM). This module plays a very important role as it is in charge of initially acquiring a topological and functional view of the network, i.e. which nodes are up and how they are interconnected, as well as which capabilities they have. NMM builds a model of the network that reflects the status and that is kept up-to-date by continuously monitoring the status and load.

It is important to note that given the SDN approach, one management function often requires the synchronous participation of several other modules. For example, to fulfil the operation of energy optimization, multiple modules including NMM, MM, AMM, TEEM and NEO are at least required to cooperate.

\section{IMPLEMENTATION OF INTELLIGENT MANAGEMENT}

In this section, three efficient network layer operations embracing joint access and backhaul network design are discussed: IP-based mobility management; network-wide energy optimization; and RANaaS placement. Joint operation of the access and the backhaul imposes specific requirements from the network layer point of view, thus, in the following subsections we demonstrate how each operation can be implemented following the designed SDN-based framework.

\section{A. IP-based mobility management}

IP-based mobility management in iJOIN follows the Distributed Mobility Management (DMM) paradigm [11] to design a flat mobility architecture introducing several anchors closer to the users. The main component of the IP-based mobility management is the anchor selection algorithm. Its goal is to select the optimal anchor for the user via which the traffic is routed outside the mobile network. This selection is based on several aspects including backhaul status, support for local breakout/offload, distributed anchoring, etc. The anchor can be the P/S-GW, a veNB, an iTN or an iLGW.

\section{1) Anchor selection algorithm}

The algorithm is formalized as a finite state machine and it is run every time an UE attaches to the network or performs a handover. The algorithm works on the network view provided by NMM module and is defined as follows:

1. If an anchor exists for the UE, then go to 4, else go to 2;

2. If the PDN connection needs mobility support, then go to 5, else go to 3;

3. If there is any local breakout node close to the UE, then go to 9 , else go to 8;

4. If the UE needs a new anchor, then go to 5, else go to 7;

5. If the attached veNB provides mobility and anchor support, then go to 6, else go to 8;

6. If the UE is expected to move much during the PDN connection lifetime, then go to 8 , else go to 9 ;

7. Maintain the same anchor;

8. Select centralized $\mathrm{P} / \mathrm{S}-\mathrm{GW}$ as anchor;

9. Select closest anchor;

\section{2) Procedure of IP-based mobility management}

As previously mentioned, AMM module is in charge of managing the IP-based mobility in the network. The approach proposed for this solution is based on the interaction of different modules. Indeed, the mobility solution is not a standalone solution and an interaction with TEEM module is mandatory. Hence, selecting the anchor for the UE is not sufficient for correctly steering the UE's data traffic and a path reconfiguration in the network is required.

a) Initial UE attachment: Upon the initial UE attachment, the AMM runs the anchor selection algorithm and computes a prioritized list of anchors. At this stage, the AMM asks TEEM to establish new paths. The AMM provides to the TEEM the veNB to which the UE is connected, the list of selected anchors and the list of selected gateways. Later, TEEM sets up a path between the veNB and the gateway to the selected anchors, starting for the most preferred anchor. Once TEEM has configured the paths, the UE is able to communicate with its peers.

b) Intra-anchor UE mobility: When the UE moves from a veNB to another, AMM evaluates whether a new anchor assignment is necessary. It may happen that no change is required, which means that the same anchor is provided back as the preferred one. Since the veNB serving the UE has changed, the AMM needs to contact the TEEM module so the forwarding path is updated in the backhaul and RAN. Consequently, AMM provides to the TEEM the new veNB to which the UE is connected, the selected anchor and the selected gateway.

c) Inter-anchor UE mobility:Instead, if a new anchor is assigned to an UE during a handover, AMM performs a two- 
step communication with TEEM. Firstly, the AMM provides to the TEEM the new veNB to which the UE is connected, the selected new anchors and the selected gateways for new anchors. In this step, the AMM requires the TEEM to compute a path between the new veNB and the new selected anchor. Secondly, the AMM provides to the TEEM the old veNB to which the UE was connected. In this step, the AMM requires the TEEM to compute a path between the new anchor and the old anchor.

\section{B. Network-wide Energy Optimization}

In order to realize improved energy efficiency over the whole network, we required intelligent algorithms. These algorithms should decide whether and how to switch on/off a network node in the running time. In addition, they must jointly consider radio access network aspects, e.g., coverage and load, and backhaul aspects, e.g., network load and latency.

\section{1) Algorithm of energy optimization}

Consider a cellular network with dense iSC deployment, which is connected to core network via backhaul transport nodes. The goal is to decrease the network power consumption by dynamically switching off one or more iSCs and iTNs during a defined time-duration of $X$ minutes. More precisely, a two-phase algorithm for total power reduction of a cellular network is implemented in the NEO module:

At first stage, an energy-efficient sub-algorithm is applied to safely switch-off access nodes, i.e. iSCs, subject to three QoE (Quality of Experience) constraints.

- Network coverage, i.e. the failure probability that a random user experiences poor signal quality when accessing the network.

- Admission control, i.e. blocking probability for flows that require a "dedicated" amount of bandwidth.

- Service delay for "best-effort" flows, that are multiplexed and have to compete for resources, i.e. the probability that the delay exceeds a desired threshold.

At the second stage, the reduction of the power-savings within the backhaul network is further investigated under two constraints.

- Flow Conversation Constraint, i.e. to ensure the capacity availability on the iTNs.

- Maximum Utilization Constraint, i.e. to confirm that each iTN can tolerate the ongoing traffic with respect to its maximum utilization.

More details about the above algorithm can be obtained from [12] and [13].

\section{2) Procedure of energy optimization}

As defined in iJOIN, the NEO module is in charge of managing the energy savings that can be achieved in the overall cellular network. Thus, the NEO module runs the above algorithm that tries to decrease the energy consumption of the whole cellular network by switching on/off iSCs and iTNs while still guaranteeing the desired user QoE.

On initialization, several kinds of operations are performed, e.g. to obtain the topological view of the network and to subscribe NEO module on NMM module in order to get updated network view timely. Also in this step, predefined parameters required by the intelligent algorithm, e.g. switchoff period and thresholds, will be initialized to proper values according to the specific deployment scenario.
After initialization, the energy optimization algorithm can be triggered periodically or by other predefined events. During the execution of the algorithm, it is determined which physical nodes, i.e. iSCs and iTNs, should be switched on or off according to the current network status.

Then the operation of switching on/off physical nodes is applied. Here such kind of operations is implemented with the transaction concept [14] in order to keep consistency among different modules, which is provided in detail as follows.

a) Switch off iSC: To switch off an iSC, the NEO module firstly initializes a transaction with AMM, TEEM, NMM included, and then requests NMM to update the network topology with the assumption that the iSC is switched off. Then NEO requests NMM to perform handover operations for all related UEs. NMM communicates with RANaaS by using southbound interface to perform UE handover one by one, where the normal handover procedure is triggered. After all handovers are completed, NEO requests NMM to switch off the iSC physically. Finally, NEO will terminate the transaction and release all related resources with all changes made so far. If the transaction fails, all changes are reverted, except for those UEs whose handover has been finished.

b) Switch on iSC: NEO module requests NMM to update the network topology and to switch on the iSC physically. Of course, all these processes are done within a transaction. And subsequent actions due to switching on an iSC such as UE handover and flow path management will be automatically processed by AMM and TEEM.

c) Switch off iTN: The process of switching off an iTN is similar to switching off an iSC except for one aspect. Instead of performing a UE handover, NEO may request AMM module to perform mobility anchor assignment for existing UEs, and request TEEM module to reconfigure paths on backhaul network for existing flows.

d) Switch on iTN: It is not much different from switching off iTN. To switch on an iTN, NEO needs to request to update network topology, re-assign mobility anchor and reconfigure flow paths. All these processes should be wrapped within a transaction.

\section{RANaaS Placement}

Obviously, different placements of RANaaS entities will impose different influences on backhaul load, cost-efficiency and resiliency within the network. To deal with the problem of RANaaS placement, the network architecture shown in Fig. 4 is defined and considered. The backhaul links of iSCs are deployed by extending the existing transport infrastructure between macro eNB and core network (e.g. C1 C4), or by newly deployed backhaul infrastructure (e.g. C5 C7).

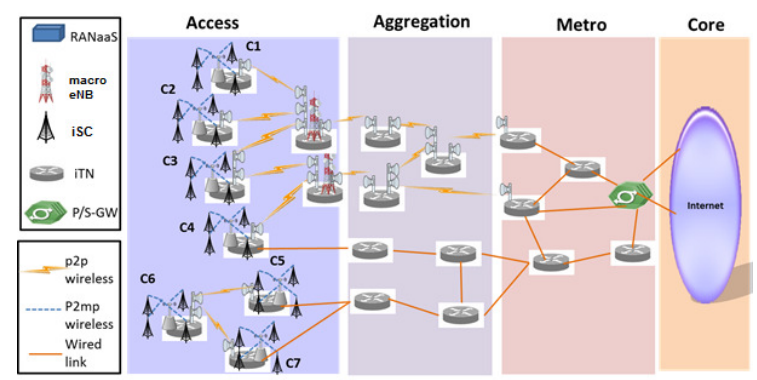

Fig. 4 Network architecture for RANaaS placement

To minimize anchors, RANaaS entities should be colocated with other data plane functions. In this study, three 
candidate locations are considered for RANaaS placement. This includes a macro eNB, an aggregating iTN and P/S-GW. A RANaaS assignment algorithm is executed periodically as a part of TEEM to optimize the assignment of RANaaS entities.

\section{1) Conceptual Analysis}

Generally, the latency increases with the number of hops between two nodes. Thus, it is obvious that the latency between RANaaS entity in P/S-GW and iSC is the largest, while the latency is the smallest when placing the RANaaS entity at the closest macro eNB.

From the viewpoint of computation resources, the more traffic is processed by a RANaaS entity, the more computation resources are required. If more iSCs are associated to one RANaaS entity, higher statistical multiplexing gains can be exploited which implies less computation resources per access point. Therefore, placing RANaaS in $\mathrm{P} / \mathrm{S}-\mathrm{GW}$ may require less computation resources than placing it at an iTN or macro eNB due to higher statistical multiplexing gains.

On the other hand, RANaaS placement has a great impact on traffic engineering where path optimization plays a key role to efficiently utilize the network resources. In the case of data offloading, data traffic may go to the internet via an iLGW. When the iLGW is placed at an iTN and the RANaaS entity is placed before the iLGW, the routing path is short. Otherwise, when the iLGW is placed at an iTN and RANaaS is placed in $\mathrm{P} / \mathrm{S}-\mathrm{GW}$, not only the routing path is sub-optimal, but also the iLGW loses its function of enabling data offloading.

\section{2) Algorithm Design}

With the iJOIN architecture, the traffic from an iSC is firstly processed by a RANaaS entity before going to the network. To optimally locate and dimension the RANaaS entities in backhaul networks, a RANaaS placement algorithm based on a genetic algorithm (GA) [15] is proposed with the objective to minimize the deployment cost.

According to the theory of genetic algorithms, a population of genomes is initialized and maintained within the predefined search space. Each genome represents a candidate solution to a given problem. A solution can be found by using techniques inspired by natural evolution, such as mutation and crossover.

a) Population initialization: The population initialization for a solution is described as follows:

1. Randomly group adjacent cells into multiple sets.

2. Randomly select a location of RANaaS entity for each set.

3. Compute paths between each cell and RANaaS entity.

4. Check the latency limitation of each path.

5. Check if lower-bound of backhaul data rate can be fulfilled for each path. If not, choose randomly another location and repeat steps 2-5 again. Otherwise, this location set of RANaaS entities is saved as one valid candidate solution.

b) Mutation: There are two ways of mutating a solution. The first one is to re-group the cells in one candidate solution. This is achieved by randomly selecting two adjacent group sets of cells and re-grouping them into two sets. The second one is to change the locations of RANaaS entities. This is done by randomly selecting a RANaaS entity and changing its location. Note that each new result has to fulfil the limitation of latency and backhaul data rate. Otherwise, the process continues until a valid candidate solution is generated.

c) Crossover: For performing crossover, two candidate solutions $S_{1}$ and $S_{2}$ are randomly selected. The crossover operation is simply creating a new solution where the association of iSC clusters to RANaaS entity is defined in solution $\mathrm{S}_{1}$ or $\mathrm{S}_{2}$.

\section{CONCLUSION}

In this paper, the network-wide operation for dense small cell deployment is investigated by taking joint optimization of backhaul and access into account. First, the concept of RANaaS and flexible function split proposed by iJOIN project is presented, which is an evolution from conventional 3GPP LTE architecture. Then the SDN-based framework for joint operation is further illustrated with logically centralized management. Following this framework, detailed implementation of IP-based mobility management, energy optimization and RANaaS placement network operations is demonstrated. Further comprehensive performance evaluation of these solutions will be carried out as the future work based on numerical simulations and/or testbeds.

\section{ACKNOWLEDGMENT}

The research leading to these results has received funding from the European Union Seventh Framework Programme (FP7/2007-2013) under grant agreement No.317941. The authors would like to acknowledge the contributions of their colleagues in iJOIN, although the views expressed are those of the authors and do not necessarily represent the project.

\section{REFERENCES}

[1] European Commission, "Digital Agenda Scoreboard", Brussels, May 2011

[2] Cisco, "Cisco Visual Networking Index: Global Mobile Data Traffic Forecast Update, 2010-2015", Cisco, February 2011

[3] "Number of available Android applications - AppBrain", http://www.appbrain.com/stats/number-of-android-apps.

[4] "Android Market Hits 10 Billion Downloads, Kicks off App Sale", http://www.wired.com/gadgetlab/2011/12/android-market-downloads/.

[5] China Mobile Research Institute, "C-RAN - Road Towards Green Radio Access Networks", in WWRF Meeting, Dusseldorf (Germany), Octeber 2011

[6] 3GPP TS 36.300 (v10.8.0), "Evolved Universal Terrestrial Radio Access (E-UTRA) and Evolved Universal Terrestrial Radio Access Network (EUTRAN); Overall description; Stage 2 (Release 10)", July 2012.

[7] P. Rost, C.J. Bernardos, A. de Domenico, M. Di Girolamo, M. Lalam, D.Sabella, and D. Wübben, "Cloud Technologies for Flexible 5G Radio Access Networks," IEEE Communications Magazine, May 2014.

[8] iJOIN, Deliverable D4.2 "Network-layer algorithms and network operation and management: candidate technologies specification," November 2014.

[9] iJOIN, Deliverable D5.2 "Final Definition of iJOIN Requirements and Scenarios," November 2014.

[10]S. Sezer, S. Scott-Hayward, P.K. Chouhan, B. Fraser, D. Lake, J. Finnegan, N. Viljoen, M. Miller and N. Rao, "Are we ready for SDN? Implementation challenges for software-defined networks", IEEE Communications Magazine, Vol.51, No.7, pp. 36-43, July 2013.

[11]Luca Cominardi, Fabio Giust, Carlos J. Bernardos, "Distributed Mobility Management for future 5G networks: overview and analysis of existing approaches", IEEE Communications Vol. 53, No.1, pp.142-149, January 2015

[12]N. Sapountzis, S. Sarantidis, T. Spyropoulos, N. Nikaein, and U. Salim, "Reducing the energy consumption of small cell networks subject to QoE constraints," IEEE GLOBECOM 2014, Austin, TX, USA, pp. 8-12, December 2014.

[13]L. Chiaraviglio, M. Mellia, and F. Neri, "Energy-aware networks: Reducing power consumption by switching off network elements," GTTI'08, Rome, Italy, May 2008.

[14]Munindar P. Singh and Michael N. Huhns, "Service-Oriented Computing: Semantics, Processes, Agents", Wiley, 2005.

[15] A. Oliveira and G.R. Mateus, "Using Genetic Algorithms to LSP Setup in MPLS Networks," Proc. XXIV Simpósio Brasileiro de Redes de Computadores, pp. 705-721, 2006. 